\title{
A CHOICE OF SOBOLEV SPACES ASSOCIATED WITH ULTRASPHERICAL EXPANSIONS
}

Jorge J. Betancor, JuAn C. Farĩ̃a, Lourdes Rodríguez-Mesa, Ricardo Testoni, AND José L. TORREA

Dedicated to the memory of Professor Carlos Segovia

Abstract

We discuss two possible definitions for Sobolev spaces associated with ultraspherical expansions. These definitions depend on the notion of higher order derivative. We show that in order to have an isomorphism between Sobolev and potential spaces, the higher order derivatives to be considered are not the iteration of the first order derivatives. Some discussions about higher order Riesz transforms are involved. Also we prove that the maximal operator for the Poisson integral in the ultraspherical setting is bounded on the Sobolev spaces.

\section{Introduction}

In this paper we introduce Sobolev spaces associated with the second order differential operator

$$
L_{\lambda}=-\frac{d^{2}}{d \theta^{2}}+\frac{\lambda(\lambda-1)}{\sin ^{2} \theta}, \quad \theta \in(0, \pi), \quad \lambda>0
$$

This expression can be factorized as

$$
L_{\lambda}=D_{\lambda}^{*} D_{\lambda}+\lambda^{2}
$$

where $D_{\lambda}=(\sin \theta)^{\lambda} \frac{d}{d \theta}(\sin \theta)^{-\lambda}$ and $D_{\lambda}^{*}$ denotes the formal adjoint of $D_{\lambda}$ in $L^{2}((0, \pi), d \theta)$.

2000 Mathematics Subject Classification. Primary: 42C05; Secondary: 42C15.

Key words. Sobolev spaces, ultraspherical expansions.

Partially supported by MTM2007/65609, MTM 2008-06621-C02-01 and PCI2006A7-0670. 
Given $\beta>0$ we consider the fractional integral $L_{\lambda}^{-\frac{\beta}{2}}$, see (6). The ultraspherical potential space, $\mathcal{L}_{\lambda, \beta}^{p}$, is defined as the range of the operator $L_{\lambda}^{-\frac{\beta}{2}}$ on the space $L^{p}(0, \pi)$. We endow $\mathcal{L}_{\lambda, \beta}^{p}$ with the norm $\|\cdot\|_{\mathcal{L}_{\lambda, \beta}^{p}}$ induced by the usual norm in $L^{p}(0, \pi)$, that is,

$$
\|f\|_{\mathcal{L}_{\lambda, \beta}^{p}}=\|g\|_{p},
$$

being $f=L_{\lambda}^{-\frac{\beta}{2}} g$ and $g \in L^{p}(0, \pi)$.

Given $m \in \mathbb{N}$, the ultraspherical Sobolev space, $W_{\lambda, m}^{p}$, is defined as follows. A function $f \in L^{p}(0, \pi)$ is in $W_{\lambda, m}^{p}$ if $D_{\lambda+k-1} \circ D_{\lambda+k-2} \circ \cdots \circ D_{\lambda}$ $f \in L^{p}(0, \pi)$, for every $k=1, \ldots, m$. Observe that

$$
D_{\lambda+k-1} \circ D_{\lambda+k-2} \circ \cdots \circ D_{\lambda}=(\sin \theta)^{\lambda+k}\left(\frac{1}{\sin \theta} \frac{d}{d \theta}\right)^{k}(\sin \theta)^{-\lambda} \text {. }
$$

We shall write in an abridge form

$$
\mathcal{D}^{(k)}=D_{\lambda+k-1} \circ D_{\lambda+k-2} \circ \cdots \circ D_{\lambda}, \quad k=1, \ldots .
$$

We make the convention $\mathcal{D}^{(0)}=\mathrm{Id}$. Along the paper the parameter $\lambda$ is a fixed number, hence we believe that the no appearance of $\lambda$ in $\mathcal{D}^{(k)}$ doesn't produce confusion to the reader.

We equipped $W_{\lambda, m}^{p}$ with the norm $\|\cdot\|_{W_{\lambda, m}^{p}}$ given by

$$
\|f\|_{W_{\lambda, m}^{p}}=\sum_{k=0}^{m}\left\|\mathcal{D}^{(k)} f\right\|_{p}, \quad f \in W_{\lambda, m}^{p} .
$$

We study some properties of the spaces $\mathcal{L}_{\lambda, m}^{p}$ and $W_{\lambda, m}^{p}$ that lead to the main result of the paper.

Theorem 1. Let $\lambda>0,1<p<\infty$ and $m \in \mathbb{N}$. Then $W_{\lambda, m}^{p}=\mathcal{L}_{\lambda, m}^{p}$.

Simplifying matters, the key behind the proof of this theorem is to establish an inequality of the type $\left\|\mathcal{D}^{(k)} f\right\|_{p} \sim\left\|L_{\lambda}^{k / 2} f\right\|_{p}$, or equivalently $\left\|\mathcal{D}^{(k)} L_{\lambda}^{-k / 2} g\right\|_{p} \sim\|g\|_{p}$. In other words, two facts have to be proved: first, the boundedness in $L^{p}(0, \pi)$ of the operators $\mathcal{D}^{(k)} L_{\lambda}^{-k / 2}$; secondly, a certain inverse process that roughly gives $\|g\|_{p} \leq\left\|\mathcal{D}^{(k)} L_{\lambda}^{-k / 2} g\right\|_{p}$. For the inverse process we use auxiliary operators, see Proposition 5. In the proof of these boundedness we use, among other tools, a Muckenhoupt multiplier transplantation result, see Lemma 1 . The operators $\mathcal{D}^{(k)} L_{\lambda}^{-k / 2}$ play the role of the "higher order Riesz transforms". These last thoughts are contained in Section 3. 
Other higher order Riesz transforms associated with the operator $L_{\lambda}$, were defined in [5]. Namely $D_{\lambda}^{k} L_{\lambda}^{-k / 2}$, where $D_{\lambda}^{k}=D_{\lambda} \circ{ }^{k} \circ \circ D_{\lambda}$ and $D_{\lambda}$ is the operator defined in (1). These Riesz transforms would suggest to define the Sobolev space as the subspace of functions $f \in L^{p}(0, \pi)$ such that $D_{\lambda}^{k} f \in L^{p}(0, \pi), k=0,1, \ldots, m$. This Sobolev space is denoted by $\mathcal{W}_{\lambda, m}^{p}$ and endowed with the norm $\|\cdot\|_{\mathcal{W}_{\lambda, m}^{p}}$ given by

$$
\|f\|_{\mathcal{W}_{\lambda, m}^{p}}=\sum_{k=0}^{m}\left\|D_{\lambda}^{k} f\right\|_{p}, \quad f \in \mathcal{W}_{\lambda, m}^{p} .
$$

In Section 4 and continuing with this line of thought, we ask if a result like Theorem 1 is possible for these Sobolev spaces, namely are the spaces $\mathcal{W}_{\lambda, m}^{p}$ and $\mathcal{L}_{\lambda, m}^{p}$ isomorphic? We prove that although the Riesz transforms, $D_{\lambda}^{k} L_{\lambda}^{-k / 2}$, are bounded in $L^{p}(0, \pi)$, the inverse process that is needed for a theorem like the Theorem 1 doesn't work. In fact the Theorem 2 below will be proved. We should mention here that we give a different proof of the boundedness of the Riesz transforms $D_{\lambda}^{k} L_{\lambda}^{-k / 2}$ from the one given in $[5]$.

Theorem 2. Let $\lambda>0,1<p<\infty$ and $m \in \mathbb{N}$. Then $W_{\lambda, m}^{p}$ is continuously contained in $\mathcal{W}_{\lambda, m}^{p}$. However $W_{\lambda, 2}^{p} \neq \mathcal{W}_{\lambda, 2}^{p}$, provided that $0<\lambda \leq 1-\frac{1}{p}$

Theorems 1 and 2 suggest that the adjective "Sobolev" has to be given to the spaces $W_{\lambda, m}^{p}$.

Finally some properties of these Sobolev spaces $W_{\lambda, m}^{p}$ are analyzed. In Section 3 it is shown that if $W_{m}^{p}(I), I$ a real interval, denotes the classical Sobolev space on $I$, then $W_{\lambda, m}^{p} \neq W_{m}^{p}(0, \pi)$, but if $f \in W_{\lambda, m}^{p}$ then $f_{\mid(a, b)} \in W_{m}^{p}(a, b)$, for every $0<a<b<\pi$ (Proposition 3). This fact and the procedure developed by Kinnunen $[\mathbf{1 1}]$, allow us to prove in Section 5 the following theorem about the maximal operator for the Poisson integral, $P_{*}^{\lambda}$.

Theorem 3. Let $\lambda>0$ and $1<p<\infty$. Then $P_{*}^{\lambda}$ is bounded from $W_{\lambda, 1}^{p}$ into itself.

Some related results for the Hermite and Laguerre families can be found in $[\mathbf{3}],[\mathbf{4}],[\mathbf{1 6}],[\mathbf{2 1}]$ and $[\mathbf{1 0}]$.

Throughout this paper by $C$ we always represent a suitable positive constant that can change in each occurrence. For every $1 \leq p<\infty$, we denote as usual by $p^{\prime}$ the conjugate exponent of $p$. 


\section{Ultraspherical potential spaces}

For every $n \in \mathbb{N}$, we denote by $\varphi_{n}^{\lambda}$ the ultraspherical function defined by

$$
\varphi_{n}^{\lambda}(\theta)=\frac{\Gamma(\lambda)}{\sqrt{\pi} 2^{\frac{1}{2}-\lambda}}\left(\frac{(n+\lambda) n !}{\Gamma(n+2 \lambda)}\right)^{\frac{1}{2}}(\sin \theta)^{\lambda} P_{n}^{\lambda}(\cos \theta), \quad \theta \in(0, \pi),
$$

where $P_{n}^{\lambda}$ is the $\lambda$-ultraspherical polynomial of degree $n$ (see $[\mathbf{2 0}]$ ). The family $\left\{\varphi_{n}^{\lambda}\right\}_{n \in \mathbb{N}}$ is an orthonormal and complete system in $L^{2}(0, \pi)$. For every $n \in \mathbb{N}, \varphi_{n}^{\lambda}$ is an eigenfunction of the operator $L_{\lambda}$, see (1), with eigenvalue $(n+\lambda)^{2}$. The system $\left\{\varphi_{n}^{\lambda}\right\}_{n \in \mathbb{N}}$ has been discussed in the literature for instance by Askey and Wainger ([1] and [2]).

Negative powers of the operator $L_{\lambda}$ can be defined as ultraspherical multipliers as follows. If $\beta>0$ the operator $L_{\lambda}^{-\beta}$ is given by

$$
L_{\lambda}^{-\beta} f=\sum_{n=0}^{\infty} \frac{a_{n}^{\lambda}(f)}{(n+\lambda)^{2 \beta}} \varphi_{n}^{\lambda}, \quad f \in L^{2}(0, \pi),
$$

where $a_{n}^{\lambda}(f)=\int_{0}^{\pi} f(\theta) \varphi_{n}^{\lambda}(\theta) d \theta$. It is clear that, for every $\beta>0, L_{\lambda}^{-\beta}$ defines a bounded operator from $L^{2}(0, \pi)$ into itself.

Let $S_{\lambda}$ be the linear space generated by the system $\left\{\varphi_{n}^{\lambda}\right\}_{n \in \mathbb{N}}$ of ultraspherical functions. This linear space plays an important role in our study. $S_{\lambda}$ is a dense subspace on $L^{p}(0, \pi), 1 \leq p<\infty$, see [19, Lemma 2.3].

Proposition 1. Let $\lambda>0, \beta>0$ and $1 \leq p<\infty$. The operator $L_{\lambda}^{-\beta}$ is bounded and one to one on $L^{p}(0, \pi)$.

Proof: It is not hard to see that, for each $f \in S_{\lambda}$

$$
L_{\lambda}^{-\beta} f(\theta)=\int_{0}^{\pi} f(\varphi) K_{\lambda, \beta}(\theta, \varphi) d \varphi, \quad \theta \in(0, \pi),
$$

where

$$
\text { (7) } K_{\lambda, \beta}(\theta, \varphi)=\frac{1}{\Gamma(2 \beta)} \int_{0}^{1} \frac{(-\log r)^{2 \beta-1}}{r} P_{r}^{\lambda}(\theta, \varphi) d r, \quad \theta, \varphi \in(0, \pi) \text {, }
$$

and the Poisson kernel $P_{r}^{\lambda}(\theta, \varphi)$ for $0<r<1$ and $\theta, \varphi \in(0, \pi)$ is given by (see $[\mathbf{1 5},(2.11)$ and $(2.12)$, p. 25])

$P_{r}^{\lambda}(\theta, \varphi)=\frac{\lambda}{\pi} r^{\lambda}\left(1-r^{2}\right) \int_{0}^{\pi} \frac{(\sin t)^{2 \lambda-1}(\sin \theta \sin \varphi)^{\lambda}}{\left(1-2 r(\cos \theta \cos \varphi+\sin \theta \sin \varphi \cos t)+r^{2}\right)^{\lambda+1}} d t$.

We can write, for $\theta, \varphi, t \in(0, \pi)$ and $0<r<1$,

(8) $1-2 r(\cos \theta \cos \varphi+\sin \theta \sin \varphi \cos t)+r^{2}$

$$
=(1-r)^{2}+2 r(1-\cos (\theta-\varphi))+2 r \sin \theta \sin \varphi(1-\cos t) .
$$


Then,

$$
P_{r}^{\lambda}(\theta, \varphi) \leq C \frac{r^{\lambda}}{(1-r)^{2 \lambda+1}}, \quad \theta, \varphi \in(0, \pi) .
$$

Moreover, by [15, (4.1)(a), p. 27],

$$
P_{r}^{\lambda}(\theta, \varphi) \leq C \frac{(1-r) r^{\lambda}}{(1-r)^{2}+(\theta-\varphi)^{2}}, \quad \theta, \varphi \in(0, \pi), \quad \frac{1}{2} \leq r<1 .
$$

From (9) and (10), it deduces that for every $\varphi \in(0, \pi)$

$$
\begin{gathered}
\int_{0}^{\pi} K_{\lambda, \beta}(\theta, \varphi) d \theta \leq C\left(\int_{0}^{\pi} \int_{\frac{1}{2}}^{1}(-\log r)^{2 \beta-1} \frac{1-r}{(1-r)^{2}+(\theta-\varphi)^{2}} d r d \theta\right. \\
\left.+\int_{0}^{\pi} \int_{0}^{\frac{1}{2}} \frac{(-\log r)^{2 \beta-1}}{r^{1-\lambda}} d r d \theta\right) \leq C
\end{gathered}
$$

By using the symmetry $K_{\lambda, \beta}(\theta, \varphi)=K_{\lambda, \beta}(\varphi, \theta)$ we get the boundedness of $L_{\lambda}^{-\beta}$ in $L^{p}((0, \pi))$ for every $1 \leq p<\infty$.

Let $n \in \mathbb{N}$. For every $f \in S_{\lambda}$ we have

$$
a_{n}^{\lambda}\left(L_{\lambda}^{-\beta} f\right)=\int_{0}^{\pi} L_{\lambda}^{-\beta}(f)(\theta) \varphi_{n}^{\lambda}(\theta) d \theta=\frac{1}{(n+\lambda)^{2 \beta}} a_{n}^{\lambda}(f), \quad n \in \mathbb{N} .
$$

As the operators $f \rightarrow \int_{0}^{\pi} L_{\lambda}^{-\beta}(f)(\theta) \varphi_{n}^{\lambda}(\theta) d \theta, f \rightarrow \int_{0}^{\pi} f(\theta) \varphi_{n}^{\lambda}(\theta) d \theta$, are bounded from $L^{p}(0, \pi)$ into $\mathbb{C}$ and $S_{\lambda}$ is dense in $L^{p}(0, \pi)$, we obtain $a_{n}^{\lambda}\left(L_{\lambda}^{-\beta} f\right)=\frac{a_{n}^{\lambda}(f)}{(n+\lambda)^{2 \beta}}, f \in L^{p}(0, \pi)$.

Suppose that $L_{\lambda}^{-\beta} f=0$, for some $f \in L^{p}(0, \pi)$. Then $a_{n}^{\lambda}(f)=0$, $n \in \mathbb{N}$. Hence, according to $[\mathbf{1 8}$, Theorem $2.2(\mathrm{~d})], f=0$. Thus, we prove that $L_{\lambda}^{-\beta}$ is one to one on $L^{p}(0, \pi)$.

Boundedness of ultraspherical multipliers has been investigated by several authors (see $[\mathbf{7}],[\mathbf{1 5}],[\mathbf{6}]$ and $[\mathbf{1 4}]$ ). In particular by using the following lemma one can prove the boundedness in $L^{p}(0, \pi), 1<p<\infty$, of $L_{\lambda}^{-\frac{m}{2}}, m \in \mathbb{N}$. This lemma is an easy consequence of $[\mathbf{1 4}$, Corollary 17.11], and it will be useful in the sequel. 
Lemma 1. Let $1<p<\infty, d \in \mathbb{Z}$ and $\lambda, \gamma>0$. Assume that

$$
g(n)=\sum_{j=0}^{J-1} c_{j}(n+1)^{-j}+O\left((n+1)^{-J}\right),
$$

where $J \geq 2(\lambda+\gamma+2)$. Then the operator

$$
T f(\theta)=\sum_{n=0}^{\infty} g(n) a_{n}^{\gamma}(f) \varphi_{n+d}^{\lambda}(\theta)
$$

is bounded in $L^{p}(0, \pi)$.

Proposition 1 gives sense to the potential spaces, $\mathcal{L}_{\lambda, \beta}^{p}$, defined in (2). It is not hard to see that $\left(\mathcal{L}_{\lambda, \beta}^{p},\|\cdot\|_{\mathcal{L}_{\lambda, \beta}^{p}}\right)$ is a Banach space. Moreover, since $S_{\lambda}$ is a dense subspace of $L^{p}(0, \pi)$ [19, Lemma 2.3], by taking into account that $L_{\lambda}^{-\frac{\beta}{2}} S_{\lambda}=S_{\lambda}$, we get that $S_{\lambda}$ is a dense subspace of $\mathcal{L}_{\lambda, \beta}^{p}$.

\section{Ultraspherical Sobolev spaces}

A standard procedure shows that the space $\left(W_{\lambda, m}^{p},\|\cdot\|_{W_{\lambda, m}^{p}}\right)$, see (5), is a Banach space. It is clear that $W_{\lambda, m+1}^{p}$ is contained in $W_{\lambda, m}^{p}$. Next we establish properties of these Sobolev spaces.

Proposition 2. Let $\lambda>0,1 \leq p<\infty$ and $m \in \mathbb{N}$. The linear space $S_{\lambda}$ is a dense subspace of $W_{\lambda, m}^{p}$.

Proof: Note firstly that, according to $[\mathbf{2 0},(4.7 .14)$, p. 81$]$, we have that

$$
\mathcal{D}^{(k)} \varphi_{n}^{\lambda}=(-1)^{k} \sqrt{\frac{\Gamma(n+1) \Gamma(n+2 \lambda+k)}{\Gamma(n-k+1) \Gamma(n+2 \lambda)}} \varphi_{n-k}^{\lambda+k}, \quad n, k \in \mathbb{N},
$$

where $\mathcal{D}^{(k)}$ is defined in (4). Here and in the sequel $\varphi_{n}^{\lambda}=0$ when $n<0$. Then, since $\lambda>0, \mathcal{D}^{(k)} \varphi_{n}^{\lambda} \in L^{p}(0, \pi), k=0,1, \ldots, m$. Hence, $S_{\lambda}$ is contained in $W_{\lambda, m}^{p}$.

Assume now that $f \in W_{\lambda, m}^{p}$. We first prove that, for every $k=$ $0,1, \ldots, m$

$$
a_{n}^{\lambda+k}\left(\mathcal{D}^{(k)} f\right)=\int_{0}^{\pi} f(\theta)\left(\mathcal{D}^{(k)}\right)^{*} \varphi_{n}^{\lambda+k}(\theta) d \theta, \quad n \in \mathbb{N},
$$

where $\left(\mathcal{D}^{(k)}\right)^{*}=(-1)^{k}(\sin \theta)^{1-\lambda}\left(\frac{1}{\sin \theta} \frac{d}{d \theta}\right)^{k}(\sin \theta)^{\lambda+k-1}$ is the formal adjoint of $\mathcal{D}^{(k)}$ in $L^{2}(0, \pi)$.

Let $n \in \mathbb{N}$ and $k=0,1, \ldots, m$. Note that for $k=0$ (13) is obvious, then suppose $k \geq 1$. We choose a sequence $\left\{\phi_{l}\right\}_{l \in \mathbb{N}}$ of smooth functions satisfying: 
(1) $\operatorname{supp} \phi_{l} \subset\left(\frac{1}{2 l}, \pi-\frac{1}{2 l}\right) ; \phi_{l}(x)=1, x \in\left(\frac{1}{l}, \pi-\frac{1}{l}\right) ; 0 \leq \phi_{l}(x) \leq 1$, for every $l \in \mathbb{N}$;

(2) For each $s \in \mathbb{N}$, there exists $C_{s}>0$ such that

$$
\left|\frac{d^{s}}{d \theta^{s}} \phi_{l}(\theta)\right| \leq C_{s} \frac{1}{\theta^{s}(\pi-\theta)^{s}}, \quad \theta \in(0, \pi) \text { and } l \in \mathbb{N} .
$$

Since $\mathcal{D}^{(k)} f \in L^{p}(0, \pi)$ and $\phi_{l}(\theta) \rightarrow 1$, as $l \rightarrow \infty$, for every $\theta \in(0, \pi)$, one has

$$
\begin{aligned}
\int_{0}^{\pi} \mathcal{D}^{(k)}(f)(\theta) \varphi_{n}^{\lambda+k}(\theta) d \theta & =\lim _{l \rightarrow \infty} \int_{0}^{\pi} \mathcal{D}^{(k)}(f)(\theta) \phi_{l}(\theta) \varphi_{n}^{\lambda+k}(\theta) d \theta \\
& =\lim _{l \rightarrow \infty} \int_{0}^{\pi} f(\theta)\left(\mathcal{D}^{(k)}\right)^{*}\left(\phi_{l}(\theta) \varphi_{n}^{\lambda+k}(\theta)\right) d \theta
\end{aligned}
$$

By using Leibniz rule we get, for every $\theta \in(0, \pi)$ and $l \in \mathbb{N}$,

$(14)$

$$
\begin{aligned}
& \left(\mathcal{D}^{(k)}\right)^{*}\left(\phi_{l}(\theta) \varphi_{n}^{\lambda+k}(\theta)\right) \\
& =(-1)^{k}(\sin \theta)^{1-\lambda}\left(\frac{1}{\sin \theta} \frac{d}{d \theta}\right)^{k}\left((\sin \theta)^{\lambda+k-1} \varphi_{n}^{\lambda+k}(\theta) \phi_{l}(\theta)\right) \\
& =(-1)^{k} \sum_{j=0}^{k}\left(\begin{array}{c}
k \\
j
\end{array}\right)(-1)^{j}(\sin \theta)^{k-j} D_{\lambda+k-j}^{*} D_{\lambda+k-(j-1)}^{*} \ldots D_{\lambda+k-1}^{*}\left(\varphi_{n}^{\lambda+k}(\theta)\right) \\
& \quad \times\left(\frac{1}{\sin \theta} \frac{d}{d \theta}\right)^{k-j}\left(\phi_{l}(\theta)\right) .
\end{aligned}
$$

Taking into account that

$$
D_{\lambda}^{*} \varphi_{n-1}^{\lambda+1}(\theta)=-\sqrt{n(n+2 \lambda)} \varphi_{n}^{\lambda}(\theta), \quad \theta \in(0, \pi),
$$

we can write, for each $\theta \in(0, \pi)$ and $j=1, \ldots, k$,

$$
\begin{aligned}
& D_{\lambda+k-j}^{*} \ldots D_{\lambda+k-1}^{*}\left(\varphi_{n}^{\lambda+k}(\theta)\right) \\
& \quad=(-1)^{j} \sqrt{\frac{\Gamma(n+j+1) \Gamma(n+2(\lambda+k))}{\Gamma(n+1) \Gamma(n+2(\lambda+k)-j)}} \varphi_{n+j}^{\lambda+k-j}(\theta) .
\end{aligned}
$$


On the other hand, a straightforward manipulation allows us to obtain

$$
\begin{aligned}
\left|\left(\frac{1}{\sin \theta} \frac{d}{d \theta}\right)^{s}\left(\phi_{l}(\theta)\right)\right| & \leq C \sum_{t=1}^{s}\left|\frac{1}{(\sin \theta)^{2 s-t}} \frac{d^{t}}{d \theta^{t}} \phi_{l}(\theta)\right| \\
& \leq C \frac{1}{(\sin \theta)^{2 s}}, \quad \theta \in(0, \pi), \quad s, l \in \mathbb{N} .
\end{aligned}
$$

By combining (14), (16), and (17) we deduce that

$\left|\left(\mathcal{D}^{(k)}\right)^{*}\left(\phi_{l}(\theta) \varphi_{n}^{\lambda+k}(\theta)\right)-\phi_{l}(\theta)\left(\mathcal{D}^{(k)}\right)^{*}\left(\varphi_{n}^{\lambda+k}(\theta)\right)\right| \leq C F_{l}(\theta), \quad \theta \in(0, \pi)$,

where $0 \leq F_{l}(\theta) \leq C, \theta \in(0, \pi)$ and $l \in \mathbb{N}$, and $\lim _{l \rightarrow \infty} F_{l}(\theta)=0$, $\theta \in(0, \pi)$.

Then, convergence dominated theorem leads to

$$
\begin{aligned}
\lim _{l \rightarrow \infty} \int_{0}^{\pi} f(\theta)\left(\mathcal{D}^{(k)}\right)^{*}\left(\phi_{l}(\theta) \varphi_{n}^{\lambda+k}(\theta)\right) d \theta & =\lim _{l \rightarrow \infty} \int_{0}^{\pi} f(\theta) \phi_{l}(\theta)\left(\mathcal{D}^{(k)}\right)^{*}\left(\varphi_{n}^{\lambda+k}(\theta)\right) d \theta \\
& =\int_{0}^{\pi} f(\theta)\left(\mathcal{D}^{(k)}\right)^{*}\left(\varphi_{n}^{\lambda+k}(\theta)\right) d \theta
\end{aligned}
$$

and (13) is proved.

By using again (16) for $j=k$, we conclude that

$$
a_{n}^{\lambda+k}\left(\mathcal{D}^{(k)} f\right)=(-1) \sqrt[k]{\frac{\Gamma(n+k+1) \Gamma(n+2(\lambda+k))}{\Gamma(n+1) \Gamma(n-k+2(\lambda+k))}} a_{n+k}^{\lambda}(f) .
$$

Let $0 \leq k \leq m$. By (12) and (18) we can write, for every $0 \leq r<1$ and $\theta \in(0, \pi)$,

$$
\begin{aligned}
\mathcal{D}^{(k)} P_{r}^{\lambda}(f)(\theta) & =\sum_{n=k}^{\infty} r^{n+\lambda} a_{n}^{\lambda}(f)(-1)^{k} \sqrt{\frac{\Gamma(n+1) \Gamma(n+2 \lambda+k)}{\Gamma(n-k+1) \Gamma(n+2 \lambda)}} \varphi_{n-k}^{\lambda+k}(\theta) \\
& =\sum_{n=k}^{\infty} r^{n+\lambda} a_{n-k}^{\lambda+k}\left(\mathcal{D}^{(k)} f\right) \varphi_{n-k}^{\lambda+k}(\theta) \\
& =\sum_{n=0}^{\infty} r^{n+\lambda+k} a_{n}^{\lambda+k}\left(\mathcal{D}^{(k)} f\right) \varphi_{n}^{\lambda+k}(\theta)=P_{r}^{\lambda+k}\left(\mathcal{D}^{(k)} f\right)(\theta)
\end{aligned}
$$

where, for every $g \in L^{p}(0, \infty), P_{r}^{\lambda}(g)$ denotes the Poisson integral of $g$ associated with the ultraspherical system $\left\{\varphi_{n}^{\lambda}\right\}_{n \in \mathbb{N}}$ and it is given by

$$
P_{r}^{\lambda}(g)(\theta)=\sum_{n=0}^{\infty} r^{n+\lambda} a_{n}^{\lambda}(g) \varphi_{n}^{\lambda}(\theta), \quad 0 \leq r<1 \text { and } \theta \in(0, \pi) .
$$


Also, for every $l \in \mathbb{N}, l>k, 0<r<1$ and $\theta \in(0, \pi)$,

(20) $\mathcal{D}^{(k)}\left(\sum_{n=l+1}^{\infty} r^{n+\lambda} a_{n}^{\lambda}(f) \varphi_{n}^{\lambda}(\theta)\right)=\sum_{n=l-k+1}^{\infty} r^{n+\lambda+k} a_{n}^{\lambda+k}\left(\mathcal{D}^{(k)} f\right) \varphi_{n}^{\lambda+k}(\theta)$.

$\left[\mathbf{1 4},(2.8)\right.$, p. 9] is used to obtain that $\left\|\varphi_{n}^{\gamma}\right\|_{q} \leq C_{\gamma, q}, n \in \mathbb{N}$, with $\gamma>0$ and $1 \leq q \leq \infty$. This justifies differentiation under the sum sign in (19) and (20) and allows us to show that

$$
\begin{aligned}
\left\|\mathcal{D}^{(k)}\left(\sum_{n=l+1}^{\infty} r^{n+\lambda} a_{n}^{\lambda}(f) \varphi_{n}^{\lambda}(\theta)\right)\right\|_{p} \\
\leq \sum_{n=l-k+1}^{\infty} r^{n+\lambda+k}\left\|\varphi_{n}^{\lambda+k}\right\|_{p}\left\|\mathcal{D}^{(k)} f\right\|_{p}\left\|\varphi_{n}^{\lambda+k}\right\|_{p^{\prime}} \\
\leq C\left\|\mathcal{D}^{(k)} f\right\|_{p} \sum_{n=l-k+1}^{\infty} r^{n+\lambda+k}, \quad 0 \leq r<1 .
\end{aligned}
$$

Let $\varepsilon>0$. According to [18, Theorem 2.2(d)] we have that

$$
P_{r}^{\lambda}(g) \rightarrow g, \text { as } r \rightarrow 1 \text {, in } L^{p}(0, \pi) .
$$

Then there exists $r_{0} \in(0,1)$ such that

$$
\left\|\mathcal{D}^{(k)}\left(P_{r_{0}}^{\lambda}(f)-f\right)\right\|_{p}<\varepsilon, \quad 0 \leq k<m
$$

and from (21) there exists $l_{0} \in \mathbb{N}$ for which

$$
\left\|\mathcal{D}^{(k)}\left(\sum_{n=l_{0}+1}^{\infty} r_{0}^{n+\lambda} a_{n}^{\lambda}(f) \varphi_{n}^{\lambda}\right)\right\|_{p}<\varepsilon, \quad 0 \leq k \leq m .
$$

Thus by (23) and (24), we conclude that

$$
\left\|\mathcal{D}^{(k)}\left(\sum_{n=0}^{l_{0}} r_{0}^{n+\lambda} a_{n}^{\lambda}(f) \varphi_{n}^{\lambda}-f\right)\right\|_{p}<2 \varepsilon, \quad 0 \leq k \leq m,
$$

that is,

$$
\left\|\sum_{n=0}^{l_{0}} r_{0}^{n+\lambda} a_{n}^{\lambda}(f) \varphi_{n}^{\lambda}-f\right\|_{W_{\lambda, m}^{p}}<2(m+1) \varepsilon .
$$

We now present some relations between our ultraspherical Sobolev spaces and the classical Sobolev spaces $W_{m}^{p}(a, b)$ on $(a, b)$ with $0 \leq a<$ $b \leq \pi$. 
Proposition 3. Let $\lambda>0,1 \leq p<\infty$ and $m \in \mathbb{N}$. Assume that $f \in W_{\lambda, m}^{p}$. Then

(i) $f \in W_{m}^{p}(a, b)$, for every $0<a<b<\pi$.

(ii) $f$ is in $W_{m}^{p}(0, \pi)$, provided that $\operatorname{supp}(f) \subset[a, b] \subset(0, \pi)$.

Moreover, if $m \geq 1$ there exist $g_{1} \in W_{m}^{p}(0, \pi) \backslash W_{\lambda, m}^{p}$ and $g_{2} \in W_{\lambda, m}^{p} \backslash$ $W_{m}^{p}(0, \pi)$, when $\lambda \leq 1-\frac{1}{p}, p>1$.

Proof: To see (i) and (ii) it is sufficient to note that if $f \in W_{\lambda, m}^{p}, \frac{d^{k}}{d \theta^{k}} f \in$ $L^{p}(K)$ for every compact subset $K$ of $(0, \pi)$ and $k=0,1, \ldots, m$.

The function $g_{1}(\theta)=1, \theta \in(0, \pi)$, is in $W_{m}^{p}(0, \pi)$ and it is not in $W_{\lambda, m}^{p}$. On the other hand $g_{2}(\theta)=(\sin \theta)^{\lambda}, \theta \in(0, \pi)$, is in $W_{\lambda, m}^{p}$. However, $\frac{d}{d \theta} g_{2}(\theta)=\lambda(\sin \theta)^{\lambda-1}(\cos \theta), \theta \in(0, \pi)$, is not in $L^{p}(0, \pi)$ when $p>1$ and $\lambda \leq 1-\frac{1}{p}$.

Our objective now is to prove Theorem 1. Previously we need to establish the boundedness of some operators.

Lemma 2. Let $\lambda>0,1 \leq p \leq \infty$ and $k \in \mathbb{N}$. Then the projector operator $P_{k}$ defined by

$$
P_{k}(f)=\sum_{n=0}^{k} a_{n}^{\lambda}(f) \varphi_{n}^{\lambda}, \quad f \in L^{p}(0, \pi),
$$

is bounded on $L^{p}(0, \pi)$.

Proof: It is sufficient to note that

$$
\left\|P_{k}(f)\right\|_{p} \leq \sum_{n=0}^{k}\left\|\varphi_{n}^{\lambda}\right\|_{p}\left\|\varphi_{n}^{\lambda}\right\|_{p^{\prime}}\|f\|_{p}, \quad f \in L^{p}(0, \pi),
$$

where $p^{\prime}$ denotes the conjugate exponent of $p$.

Proposition 4. Let $\lambda>0,1<p<\infty$ and $k \in \mathbb{N}$. We define the operator $R_{\lambda, 1}^{k}$ on $S_{\lambda}$ by

$$
R_{\lambda, 1}^{k} f=\mathcal{D}^{(k)} L_{\lambda}^{-\frac{k}{2}} f, \quad f \in S_{\lambda} .
$$

Then $R_{\lambda, 1}^{k}$ can be extended to $L^{p}(0, \pi)$ as a bounded operator from $L^{p}(0, \pi)$ into itself.

Proof: According to (12) we can write

$$
R_{\lambda, 1}^{k} f=\sum_{n=k}^{\infty}(-1) \sqrt[k]{\frac{\Gamma(n+1) \Gamma(n+2 \lambda+k)}{\Gamma(n+1-k) \Gamma(n+2 \lambda)}} \frac{1}{(n+\lambda)^{k}} a_{n}^{\lambda}(f) \varphi_{n-k}^{\lambda+k}, \quad f \in S_{\lambda} .
$$


We denote by $g$ the function

$$
g(n)=(-1)^{k} \sqrt{\frac{\Gamma(n+1) \Gamma(n+2 \lambda+k)}{\Gamma(n+1-k) \Gamma(n+2 \lambda)}} \frac{1}{(n+\lambda)^{k}}, \quad n=k, k+1, \ldots .
$$

It is clear that $g(n)=(-1)^{k} \sqrt{\frac{p(n)}{(n+\lambda)^{2 k}}}, n=k, k+1, \ldots$, where $p$ is a polynomial with degree $2 k$. Let us write $g(z)=(-1)^{k} \sqrt{\frac{p(z)}{(z+\lambda)^{2 k}}},|z|>k$, and consider the function $h(z)=g\left(\frac{1-z}{z}\right),|z|<\delta$, for some $\delta>0$ small enough. Then

$$
h(z)=(-1)^{k} \sqrt{\frac{p\left(\frac{1-z}{z}\right) z^{2 k}}{(1+(\lambda-1) z)^{2 k}}}, \quad|z|<\delta .
$$

Since $\lim _{z \rightarrow 0} p\left(\frac{1-z}{z}\right) z^{2 k}=1$ and $p\left(\frac{1-z}{z}\right) z^{2 k}$ is a polynomial, $h$ is holomorphic in $\{z \in \mathbb{C}:|z|<\delta\}$, when $\delta$ is small enough. Then, for every $l \in \mathbb{N}$,

$$
g(n)=\sum_{j=0}^{l} c_{j} \frac{1}{(n+1)^{j}}+\mathcal{O}\left(\frac{1}{(n+1)^{l+1}}\right)
$$

when $n \geq n_{0}=n_{0}(\lambda, k)$, for a certain $n_{0} \in \mathbb{N}$. We define $g_{J}, J \in \mathbb{N}$, by

$$
g_{J}(n)= \begin{cases}\sum_{j=0}^{J-1} c_{j} \frac{1}{(n+1)^{j}}, & 0<n<n_{0} \\ g(n), & n \geq n_{0} .\end{cases}
$$

Let us choose $J \geq 2(2 \lambda+k+2)$. Applying Lemma 1, we get that the operator $R_{\lambda, 1, J}^{k}$ defined by

$$
R_{\lambda, 1, J}^{k} f=\sum_{n=k}^{\infty} g_{J}(n) a_{n}^{\lambda}(f) \varphi_{n-k}^{\lambda+k}, \quad f \in S_{\lambda},
$$

can be extended to $L^{p}(0, \pi)$ as a bounded operator.

Also, by proceeding as in the proof of Lemma 2 we can establish that the operator $R_{\lambda, 1}^{k}-R_{\lambda, 1, J}^{k}$ is a bounded operator from $L^{p}(0, \pi)$ into itself.

Thus, we conclude that $R_{\lambda, 1}^{k}$ can be extended to $L^{p}(0, \pi)$ as a bounded operator from $L^{p}(0, \pi)$ into itself. 
Proposition 5. Let $\lambda>0,1<p<\infty$ and $k \in \mathbb{N}$. We define on $S_{\lambda+k}$ the operator

$$
R_{\lambda, 2}^{k} f=\left(\mathcal{D}^{(k)}\right)^{*} L_{\lambda+k}^{-\frac{k}{2}} f, \quad f \in S_{\lambda+k} .
$$

Then, $R_{\lambda, 2}^{k}$ can be extended to $L^{p}(0, \pi)$ as a bounded operator from $L^{p}(0, \pi)$ into itself.

Proof: Let $f \in S_{\lambda+k}$. According to (16) for $j=k$, we have that

$$
R_{\lambda, 2}^{k} f=\sum_{n=0}^{\infty} \frac{(-1)^{k}}{(n+\lambda+k)^{k}} \sqrt{\frac{\Gamma(n+2 \lambda+2 k) \Gamma(n+k+1)}{\Gamma(n+k+2 \lambda) \Gamma(n+1)}} a_{n}^{\lambda+k}(f) \varphi_{n+k}^{\lambda} .
$$

By proceeding as in the proof of Proposition 4 we can see that $R_{\lambda, 2}$ can be extended to $L^{p}(0, \pi)$ as a bounded operator from $L^{p}(0, \pi)$ into itself.

We can write, for every $f \in S_{\lambda}$,

$$
R_{\lambda, 2}^{k} R_{\lambda, 1}^{k} f=\sum_{n=k}^{\infty} \frac{\Gamma(n+2 \lambda+k) \Gamma(n+1)}{\Gamma(n+2 \lambda) \Gamma(n-k+1)} \frac{1}{(\lambda+n)^{2 k}} a_{n}^{\lambda}(f) \varphi_{n}^{\lambda} .
$$

In the next proposition we prove the boundedness of the inverse of $R_{\lambda, 2}^{k} R_{\lambda, 1}^{k}$ on the linear space $S_{\lambda, k}=\left\{f \in S_{\lambda}: a_{n}^{\lambda}(f)=0, n=0,1, \ldots, k-\right.$ $1\}$.

Proposition 6. Let $\lambda>0,1<p<\infty$ and $k \in \mathbb{N}$. The operator $T_{\lambda}^{k}$ defined by

$$
T_{\lambda}^{k} f=\sum_{n=k}^{\infty} \frac{\Gamma(n+2 \lambda) \Gamma(n-k+1)(\lambda+n)^{2 k}}{\Gamma(n+2 \lambda+k) \Gamma(n+1)} a_{n}^{\lambda}(f) \varphi_{n}^{\lambda}, \quad f \in S_{\lambda},
$$

can be extended as a bounded operator from $L^{p}(0, \pi)$ into itself.

Proof: Fix $J \geq 4(\lambda+1)$. By proceeding as in the proof of Proposition 4 we first take a function $g_{J}$ such that

$$
g_{J}(n)=\frac{\Gamma(n+2 \lambda) \Gamma(n-k+1)(\lambda+n)^{2 k}}{\Gamma(n+2 \lambda+k) \Gamma(n+1)},
$$

for $n$ large enough. In order to establish the boundedness property for the operator $T_{\lambda}^{k}$, it is then sufficient to prove that the operator

$$
T_{\lambda, J}^{k} f=\sum_{n=k}^{\infty} g_{J}(n) a_{n}^{\lambda}(f) \varphi_{n}^{\lambda}, \quad f \in S_{\lambda},
$$


can be extended to $L^{p}(0, \pi)$ into itself. Moreover, Lemma 2 allows us to reduce the boundedness of $T_{\lambda, J}^{k}$ to functions $f \in S_{\lambda, k}$. Indeed, suppose that

$$
\left\|T_{\lambda, J}^{k} f\right\|_{p} \leq C\|f\|_{p}, \quad f \in S_{\lambda, k} .
$$

Let $f \in S_{\lambda}$. We write $f=f_{0}+f_{1}$, where $f_{0}=P_{k-1} f$. Then

$$
T_{\lambda, J} f=T_{\lambda, J}^{k} f_{0}+T_{\lambda . J}^{k} f_{1}=T_{\lambda, J}^{k} f_{1} .
$$

Since $f_{1} \in S_{\lambda, k}$, by (25) and Lemma 2 we get that

$$
\left\|T_{\lambda, J}^{k} f\right\|_{p}=\left\|T_{\lambda, J}^{k} f_{1}\right\|_{p} \leq C\left\|f_{1}\right\|_{p} \leq C\left(\|f\|_{p}+\left\|f_{0}\right\|_{p}\right) \leq C\|f\|_{p} .
$$

Finally, to prove the boundedness of the operator $T_{\lambda, J}^{k}$ on $S_{\lambda, k}$ we apply Lemma 1.

Proof of Theorem 1: $S_{\lambda}$ is a dense subspace of $W_{\lambda, m}^{p}$ (Proposition 2) and of $\mathcal{L}_{\lambda, m}^{p}\left(\left[\mathbf{1 9}\right.\right.$, Lemma 2.3]). Moreover, if $\left(f_{n}\right)_{n=1}^{\infty}$ is a sequence such that $f_{n} \rightarrow f$, as $n \rightarrow \infty$, in $W_{\lambda, m}^{p}$ or in $\mathcal{L}_{\lambda, m}^{p}$, then $f_{n} \rightarrow f$, as $n \rightarrow \infty$, in $L^{p}(0, \pi)$. Hence, to see that $W_{\lambda, m}^{p}=\mathcal{L}_{\lambda, m}^{p}$ it is sufficient to show that there exists $C>0$ such that

$$
\frac{1}{C}\|f\|_{W_{\lambda, m}^{p}} \leq\|f\|_{\mathcal{L}_{\lambda, m}^{p}} \leq C\|f\|_{W_{\lambda, m}^{p}}, \quad f \in S_{\lambda} .
$$

Assume that $f, g \in S_{\lambda}$ such that $f=L_{\lambda}^{-\frac{m}{2}} g$. We write $g=g_{1}+g_{2}$, where $g_{1}=\sum_{n=0}^{m-1} a_{n}^{\lambda}(g) \varphi_{n}^{\lambda}=\sum_{n=0}^{m-1}(n+\lambda)^{m} a_{n}^{\lambda}(f) \varphi_{n}^{\lambda}$. Then, according to Propositions 5 and 6 , since $R_{\lambda, 1}^{m} g_{2}=R_{\lambda, 1}^{m} g$, we get

$$
\begin{aligned}
\|f\|_{\mathcal{L}_{\lambda, m}^{p}} & =\|g\|_{p} \leq\left\|g_{1}\right\|_{p}+\left\|g_{2}\right\|_{p} \\
& \leq \sum_{n=0}^{m-1}(n+\lambda)^{m}\left\|\varphi_{n}^{\lambda}\right\|_{p}\left\|\varphi_{n}^{\lambda}\right\|_{p^{\prime}}\|f\|_{p}+\left\|T_{\lambda}^{m} R_{\lambda, 2}^{m} R_{\lambda, 1}^{m} g_{2}\right\|_{p} \\
& \leq\|f\|_{p} \sum_{n=0}^{m-1}(n+\lambda)^{m}\left\|\varphi_{n}^{\lambda}\right\|_{p}\left\|\varphi_{n}^{\lambda}\right\|_{p^{\prime}}+\left\|T_{\lambda}^{m} R_{\lambda, 2}^{m} R_{\lambda, 1}^{m} g\right\|_{p} \\
& \leq C\left(\|f\|_{p}+\left\|R_{\lambda, 1}^{m} g\right\|_{p}\right) \leq C\left(\|f\|_{p}+\left\|\mathcal{D}^{(m)} L_{\lambda}^{-\frac{m}{2}} g\right\|_{p}\right) \\
& \leq C\left(\|f\|_{p}+\left\|\mathcal{D}^{(m)} f\right\|_{p}\right) \leq C\|f\|_{W_{\lambda, m}^{p}} .
\end{aligned}
$$


On the other hand, Propositions 4 and 1 lead to

$$
\begin{aligned}
\|f\|_{W_{\lambda, m}^{p}} & =\sum_{k=0}^{m}\left\|\mathcal{D}^{(k)} f\right\|_{p}=\sum_{k=0}^{m}\left\|\mathcal{D}^{(k)} L_{\lambda}^{-\frac{m}{2}} g\right\|_{p}=\sum_{k=0}^{m}\left\|R_{\lambda, 1}^{k} L^{-\frac{m-k}{2}} g\right\|_{p} \\
& \leq C \sum_{k=0}^{m}\left\|L^{-\frac{m-k}{2}} g\right\|_{p} \leq C\|g\|_{p}=C\|f\|_{\mathcal{L}_{\lambda, m}^{p}} .
\end{aligned}
$$

Thus (26) is established.

\section{Alternative definition of Sobolev spaces}

As we said in the introduction the $n$-th order Riesz transform associated with the operator $L_{\lambda}$ is given by

$$
\mathcal{R}_{\lambda}^{k} f=D_{\lambda}^{k} L_{\lambda}^{-\frac{k}{2}} f, \quad f \in S_{\lambda} .
$$

We observe that $D_{\lambda}^{k}=D_{\lambda} \circ \cdots \cdot{ }^{k} \circ D_{\lambda}=(\sin \theta)^{\lambda} \frac{d^{k}}{d \theta^{k}}(\sin \theta)^{-\lambda}$, for every $k \in$ $\mathbb{N}$. By using Propositions 4 and 5 we will prove that $\mathcal{R}_{\lambda}^{k}$ can be extended to $L^{p}(0, \pi)$ as a bounded operator, for every $k \in \mathbb{N}$ and $1<p<\infty$. As it was mentioned our proof is different from the one presented in [5].

Proposition 7. Let $\lambda>0, k \in \mathbb{N}$ and $1<p<\infty$. Then $\mathcal{R}_{\lambda}^{k}$ defines a bounded operator from $L^{p}(0, \pi)$ into itself.

Proof: We denote by $\Lambda=\frac{1}{\sin \theta} \frac{d}{d \theta}$. A carefull analysis shows that, for every $k \in \mathbb{N}$,

$$
\frac{d^{k}}{d \theta^{k}}=\sum_{\ell=1}^{k} p_{\ell, k}(\theta)(\sin \theta)^{\ell} \Lambda^{\ell}
$$

where

$$
p_{\ell, k}(\theta)= \begin{cases}\sum_{m=0}^{N_{\ell, k}} c_{m}^{\ell, k}\left(\frac{\cos \theta}{\sin \theta}\right)^{2 m}, & \text { when } \ell+k \text { is even }, \\ \sum_{m=0}^{N_{\ell, k}} d_{m}^{\ell, k}\left(\frac{\cos \theta}{\sin \theta}\right)^{2 m+1}, & \text { when } \ell+k \text { is odd }\end{cases}
$$

for certain constants $c_{m}^{\ell, k}$ and $d_{m}^{\ell, k}$. Here, $N_{\ell, k}$ depends on $\ell$ and $k$ as it is indicated in the following table: 


\begin{tabular}{|c|c|c|c|c|}
\cline { 2 - 5 } \multicolumn{1}{c|}{} & \multicolumn{2}{c|}{$k$ even } & \multicolumn{2}{c|}{$k$ odd } \\
\cline { 2 - 5 } \multicolumn{1}{c|}{} & $1 \leq \ell \leq \frac{k}{2}$ & $\frac{k}{2}<\ell \leq k$ & $1 \leq \ell \leq\left[\frac{k}{2}\right]+1$ & {$\left[\frac{k}{2}\right]+1<\ell \leq k$} \\
\hline$\ell$ even & {$\left[\frac{\ell}{2}\right]$} & $\frac{k}{2}-\left[\frac{\ell}{2}\right]$ & {$\left[\frac{\ell}{2}\right]-1$} & {$\left[\frac{k}{2}\right]-\left[\frac{\ell}{2}\right]$} \\
\hline$\ell$ odd & {$\left[\frac{\ell}{2}\right]$} & $\frac{k}{2}-\left[\frac{\ell}{2}\right]-1$ & {$\left[\frac{\ell}{2}\right]$} & {$\left[\frac{k}{2}\right]-\left[\frac{\ell}{2}\right]$} \\
\hline
\end{tabular}

Hence, for every $\theta \in(0, \pi)$,

(28) $\left|p_{\ell, k}(\theta)\right| \leq C(\sin \theta)^{-\frac{k}{2}+\left|\ell-\frac{k}{2}\right|}, \quad$ if $k$ is even and $1 \leq \ell \leq k$,

and

(29) $\left|p_{\ell, k}(\theta)\right| \leq C(\sin \theta)^{-\left[\frac{k}{2}\right]+\left|\ell-\left[\frac{k}{2}\right]-1\right|}, \quad$ if $k$ is odd and $1 \leq \ell \leq k$.

Let $f \in S_{\lambda}$. From (27) and taking into account (12) and (18), it deduces that, for every $k \in \mathbb{N}$,

$(30)$

$$
\begin{aligned}
D_{\lambda}^{k} L_{\lambda}^{-\frac{k}{2}} f(\theta) & =\sum_{\ell=1}^{k} p_{\ell, k}(\theta) \mathcal{D}^{(\ell)} L_{\lambda}^{-\frac{k}{2}} f(\theta) \\
& =\sum_{\ell=1}^{k} p_{\ell, k}(\theta) \mathcal{D}^{(\ell)} L_{\lambda}^{-\frac{k-\ell}{2}} L_{\lambda}^{-\frac{\ell}{2}} f(\theta) \\
& =\sum_{\ell=1}^{k} p_{\ell, k}(\theta) L_{\lambda+\ell}^{-\frac{k-\ell}{2}} \mathcal{D}^{(\ell)} L_{\lambda}^{-\frac{\ell}{2}} f(\theta) \\
& =\sum_{\ell=1}^{k} p_{\ell, k}(\theta) L_{\lambda+\ell}^{-\frac{k-\ell}{2}} R_{\lambda, 1}^{\ell} f(\theta), \quad \theta \in(0, \pi) .
\end{aligned}
$$

By using estimations (28) and (29) we then obtain that, for each $\theta \in$ $(0, \pi)$

$$
\begin{aligned}
\left|D_{\lambda}^{k} L_{\lambda}^{-\frac{k}{2}} f(\theta)\right| \leq C\left(\sum_{\ell=1}^{\frac{k}{2}} \mid\right. & \frac{1}{(\sin \theta)^{\ell}} L_{\lambda+\ell}^{-\frac{\ell}{2}} L_{\lambda+\ell}^{-\frac{k-2 \ell}{2}} R_{\lambda, 1}^{\ell} f(\theta) \mid \\
& \left.+\sum_{\ell=\frac{k}{2}+1}^{k}\left|\frac{1}{(\sin \theta)^{k-\ell}} L_{\lambda+\ell}^{-\frac{k-\ell}{2}} R_{\lambda, 1}^{\ell} f(\theta)\right|\right),
\end{aligned}
$$


when $k$ is even, and

$$
\begin{array}{r}
\left|D_{\lambda}^{k} L_{\lambda}^{-\frac{k}{2}} f(\theta)\right| \leq C\left(\sum_{\ell=1}^{\left[\frac{k}{2}\right]+1}\left|\frac{1}{(\sin \theta)^{\ell-1}} L_{\lambda+\ell}^{-\frac{\ell-1}{2}} L_{\lambda+\ell}^{-\frac{k-2 \ell+1}{2}} R_{\lambda, 1}^{\ell} f(\theta)\right|\right. \\
\left.+\sum_{\ell=\left[\frac{k}{2}\right]+2}^{k}\left|\frac{1}{(\sin \theta)^{k-\ell}} L_{\lambda+\ell}^{-\frac{k-\ell}{2}} R_{\lambda, 1}^{\ell} f(\theta)\right|\right),
\end{array}
$$

in the case that $k$ is odd.

Hence, according to Propositions 1 and 4, the boundedness of the operator $\mathcal{R}_{\lambda}^{(k)}$ will be established when we prove that, for every $\lambda>0$ and $j \in \mathbb{N}$, the operator

$$
C_{\lambda, j} f=\frac{1}{(\sin \theta)^{j}} L_{\lambda+j}^{-\frac{j}{2}} f
$$

is bounded from $L^{p}(0, \pi)$ into itself. We proceed by induction on $j$. Indeed, let $\lambda>0$ and $1<p<\infty$. Note firstly that

$$
\begin{aligned}
R_{\lambda, 2}^{1} f & =D_{\lambda}^{*} L_{\lambda+1}^{-\frac{1}{2}} f=-(\sin \theta)^{-\lambda} \frac{d}{d \theta}\left((\sin \theta)^{\lambda} L_{\lambda+1}^{-\frac{1}{2}} f\right) \\
& =-(\sin \theta)^{-\lambda} \frac{d}{d \theta}\left((\sin \theta)^{2 \lambda+1}(\sin \theta)^{-\lambda-1} L_{\lambda+1}^{-\frac{1}{2}} f\right) \\
& =-(2 \lambda+1) \frac{\cos \theta}{\sin \theta} L_{\lambda+1}^{-\frac{1}{2}} f-(\sin \theta)^{\lambda+1} \frac{d}{d \theta}\left((\sin \theta)^{-\lambda-1} L_{\lambda+1}^{-\frac{1}{2}} f\right) \\
& =-(2 \lambda+1) \frac{\cos \theta}{\sin \theta} L_{\lambda+1}^{-\frac{1}{2}} f-R_{\lambda+1,1}^{1} f, \quad f \in S_{\lambda+1} .
\end{aligned}
$$

Thus, by Propositions 4 and 5 the operator $f \rightarrow \frac{\cos \theta}{\sin \theta} L_{\lambda+1}^{-\frac{1}{2}} f$ is bounded from $L^{p}(0, \pi)$ into itself. Also, by Proposition $1, L_{\lambda+1}^{-\frac{1}{2}}$ is bounded on $L^{p}(0, \pi)$. Then

$$
\begin{aligned}
\int_{0}^{\pi}\left|\frac{1}{\sin \theta} L_{\lambda+1}^{-\frac{1}{2}} f(\theta)\right|^{p} d \theta \leq & C\left(\int_{\frac{\pi}{4}}^{\frac{3 \pi}{4}}\left|L_{\lambda+1}^{-\frac{1}{2}} f(\theta)\right|^{p} d \theta\right. \\
& \left.+\left(\int_{0}^{\frac{\pi}{4}}+\int_{\frac{3 \pi}{4}}^{\pi}\right)\left|\frac{\cos \theta}{\sin \theta} L_{\lambda+1}^{-\frac{1}{2}} f(\theta)\right|^{p} d \theta\right) \\
\leq & C\|f\|_{p}^{p}, \quad f \in L^{p}(0, \pi) .
\end{aligned}
$$

Hence $C_{\lambda, 1}$ is bounded from $L^{p}(0, \pi)$ into $L^{p}(0, \pi)$. 
Suppose now that the operator $C_{\lambda, j}$ is bounded on $L^{p}(0, \pi)$, for every $\lambda>0$ and $j=0, \ldots, s$, where $s \in \mathbb{N}$. Let us see that the operator $C_{\lambda, s+1}$ is bounded on $L^{p}(0, \pi)$, with $\lambda>0$ fixed. By using Leibniz rule it follows that, for every $f \in S_{\lambda+s+1}$,

(31)

$$
\begin{aligned}
R_{\lambda, 2}^{s+1} f= & \left(\mathcal{D}^{(s+1)}\right)^{*} L_{\lambda+s+1}^{-\frac{s+1}{2}} f \\
= & (-1)^{s+1}(\sin \theta)^{-\lambda+1}\left(\frac{1}{\sin \theta} \frac{d}{d \theta}\right)^{s+1}\left((\sin \theta)^{\lambda+s} L_{\lambda+s+1}^{-\frac{s+1}{2}} f\right) \\
= & (-1)^{s+1} \sum_{j=1}^{s+1}\left(\begin{array}{c}
s+1 \\
j
\end{array}\right)(\sin \theta)^{-2 \lambda-s-j}\left(\frac{1}{\sin \theta} \frac{d}{d \theta}\right)^{s+1-j} \\
& \times\left((\sin \theta)^{2(\lambda+s)+1}\right) D_{\lambda+s+j} \circ \cdots \circ D_{\lambda+s+1} L_{\lambda+s+1}^{-\frac{s+1}{2}} f+\mathcal{T}_{\lambda, s} f,
\end{aligned}
$$

where the operator $\mathcal{T}_{\lambda, s}$ is defined by

$$
\mathcal{T}_{\lambda, s} f=(-1)^{s+1}(\sin \theta)^{-2 \lambda-s}\left(\frac{1}{\sin \theta} \frac{d}{d \theta}\right)^{s+1}\left((\sin \theta)^{2(\lambda+s)+1}\right) L_{\lambda+s+1}^{-\frac{s+1}{2}} f .
$$

We observe that (12) and (18) lead to

$D_{\lambda+s+j} \circ \cdots \circ D_{\lambda+s+1} L_{\lambda+s+1}^{-\frac{s+1}{2}} f=L_{\lambda+s+1+j}^{-\frac{s+1-j}{2}} R_{\lambda+s+1,1}^{j} f, \quad j=1, \ldots, s+1$.

Also, a straightforward manipulation shows that

$$
\begin{aligned}
& \sum_{j=1}^{s+1}\left(\begin{array}{c}
s+1 \\
j
\end{array}\right)(\sin \theta)^{-2 \lambda-s-j}\left(\frac{1}{\sin \theta} \frac{d}{d \theta}\right)^{s+1-j} \\
& \times\left((\sin \theta)^{2(\lambda+s)+1}\right) L_{\lambda+s+1+j}^{-\frac{s+1-j}{2}} R_{\lambda+s+1,1}^{j} f \mid \\
& \leq C \sum_{j=1}^{s+1}\left|\frac{1}{(\sin \theta)^{1+s-j}} L_{\lambda+2 j+s+1-j}^{-\frac{s+1-j}{2}} R_{\lambda+s+1,1}^{j} f\right| .
\end{aligned}
$$


From this estimation, and by using the induction hypothesis and Proposition 4 we deduce that the operator

$$
\begin{aligned}
f \rightarrow \sum_{j=1}^{s+1}\left(\begin{array}{c}
s+1 \\
j
\end{array}\right)(\sin \theta)^{-2 \lambda-s-j} & \left(\frac{1}{\sin \theta} \frac{d}{d \theta}\right)^{s+1-j} \\
& \times\left((\sin \theta)^{2(\lambda+s)+1}\right) L_{\lambda+s+1+j}^{-\frac{s+1-j}{2}} R_{\lambda+s+1,1}^{j} f
\end{aligned}
$$

is bounded from $L^{p}(0, \pi)$ into itself.

Hence, from (31) and Proposition 5, it follows that the operator $\mathcal{T}_{\lambda, s}$ is bounded from $L^{p}(0, \pi)$ into itself. We can write

$$
(\sin \theta)^{-2 \lambda-s}\left(\frac{1}{\sin \theta} \frac{d}{d \theta}\right)^{s+1}\left((\sin \theta)^{2(\lambda+s)+1}\right)=\frac{q(\theta)}{(\sin \theta)^{s+1}},
$$

where $\theta \in(0, \pi), q \in C^{\infty}(\mathbb{R}), q(0) \neq 0$ and $q(\pi) \neq 0$. By choosing $\delta>0$ such that $q(\theta) \neq 0$ for $\theta \in[0, \delta] \cup[\pi-\delta, \pi]$ we have that

$$
\begin{aligned}
\int_{0}^{\pi}\left|C_{\lambda, s+1} f(\theta)\right|^{p} d \theta \leq & \left(\int_{0}^{\delta}+\int_{\pi-\delta}^{\pi}\right)\left|\frac{1}{q(\theta)} \mathcal{T}_{\lambda, s} f(\theta)\right|^{p} d \theta \\
& +C \int_{\delta}^{\pi-\delta}\left|L_{\lambda+s+1}^{-\frac{s+1}{2}} f(\theta)\right|^{p} d \theta \\
\leq & C\left(\int_{0}^{\pi}\left|\mathcal{T}_{\lambda, s} f(\theta)\right|^{p} d \theta+\int_{0}^{\pi}\left|L_{\lambda+s+1}^{-\frac{s+1}{2}} f(\theta)\right|^{p} d \theta\right) \\
\leq & C\|f\|_{p}^{p},
\end{aligned}
$$

for $f \in L^{p}(0, \pi)$. Hence $C_{\lambda, s+1}$ is bounded on $L^{p}(0, \pi)$.

Thus, the proof is finished.

Finally we can give the proof of Theorem 2, see Introduction, which establishes the relation between the spaces $W_{\lambda, m}^{p}$ and $\mathcal{W}_{\lambda, m}^{p}$.

Proof of Theorem 2: Assume that $f \in W_{\lambda, m}^{p}$. By Theorem 1 there exists $g \in L^{p}(0, \pi)$ such that $f=L_{\lambda}^{-\frac{m}{2}} g$ and $\|g\|_{p}$ is equivalent to $\|f\|_{W_{\lambda, m}^{p}}$. Then, by Propositions 7 and 1 , for every $k=0,1, \ldots, m$, we have that $\left\|D_{\lambda}^{k} f\right\|_{p}=\left\|D_{\lambda}^{k} L_{\lambda}^{-\frac{m}{2}} g\right\|_{p}=\left\|\mathcal{R}_{\lambda}^{k} L_{\lambda}^{-\frac{m-k}{2}} g\right\|_{p} \leq C\left\|L_{\lambda}^{-\frac{m-k}{2}} g\right\|_{p} \leq C\|f\|_{W_{\lambda, m}^{p}}$.

Thus we conclude that $W_{\lambda, m}^{p}$ is continuously contained in $\mathcal{W}_{\lambda, m}^{p}$. 
It is not difficult to see that, if $0<\lambda \leq 1-\frac{1}{p}$, the function $f(\theta)=$ $\theta(\sin \theta)^{\lambda}$ is in $\mathcal{W}_{\lambda, 2}^{p}$ but not in $W_{\lambda, 2}^{p}$.

\section{A maximal operator on the ultraspherical Sobolev spaces}

Kinnunen [11] (see also [12] and [13]) proved that the Hardy-Littlewood maximal operator is bounded in the classical Sobolev space $W_{1}^{p}\left(\mathbb{R}^{n}\right)$ for every $1<p<\infty$. In this section we prove that the maximal operator associated with the Poisson integral for the operator $L_{\lambda}$ is bounded from $W_{\lambda, 1}^{p}$ into itself, for every $1<p<\infty$.

The maximal operator associated with $\left\{P_{r}^{\lambda}\right\}_{0 \leq r<1}$ is defined by

$$
P_{*}^{\lambda}(f)=\sup _{0 \leq r<1}\left|P_{r}^{\lambda}(f)\right| .
$$

This operator $P_{*}^{\lambda}$ is bounded from $L^{p}(0, \pi)$ into itself, for every $1<p<$ $\infty$ and from $L^{1}(0, \pi)$ into $L^{1, \infty}(0, \pi)[\mathbf{1 8}$, Theorem 2.2]. Now we can give the proof of Theorem 3 .

Proof: Let $f \in W_{\lambda, 1}^{p}$ and let $\left\{r_{j}\right\}_{j=1}^{\infty}$ be an enumeration of the rational numbers in $(0,1)$. Then, we have

$$
P_{*}^{\lambda}(f)(\theta)=\sup _{j \in \mathbb{N}}\left|P_{r_{j}}^{\lambda}(f)(\theta)\right|=\sup _{k \in \mathbb{N}} \max _{1 \leq j \leq k}\left|P_{r_{j}}^{\lambda}(f)(\theta)\right|, \quad \theta \in(0, \pi) .
$$

Let $j \in \mathbb{N}$. According to $\left[\mathbf{1 5}\right.$, Theorem 3], $P_{r_{j}}^{\lambda}(f) \in C^{\infty}(0, \pi)$. Moreover, by (19)

$$
D_{\lambda} P_{r_{j}}^{\lambda}(f)(\theta)=P_{r_{j}}^{\lambda+1}\left(D_{\lambda}(f)\right)(\theta), \quad \theta \in(0, \pi) .
$$

Then, by using [18, Theorem 2.2], we conclude that $P_{r_{j}}^{\lambda}(f) \in W_{\lambda, 1}^{p}$. Hence, $P_{r_{j}}^{\lambda}(f) \in W_{1}^{p}(a, b)$, for every $0<a<b<\pi$ (see Proposition 3).

By [8, Lemma 7.6] we get that, for every $k \in \mathbb{N}$ and $0<a<b<\pi$,

$$
\begin{array}{r}
\left|\frac{d}{d \theta} \max _{1 \leq j \leq k}\right|(\sin \theta)^{-\lambda} P_{r_{j}}^{\lambda}(f)(\theta)|| \leq \max _{1 \leq j \leq k}\left|\frac{d}{d \theta}\right|(\sin \theta)^{-\lambda} P_{r_{j}}^{\lambda}(f)(\theta)||, \\
\text { a.e. } \quad \theta \in(0, \pi) .
\end{array}
$$

Hence

$$
\begin{array}{r}
\left|D_{\lambda}\left(\max _{1 \leq j \leq k}\left|P_{r_{j}}^{\lambda}(f)(\theta)\right|\right)\right| \leq \max _{1 \leq j \leq k}\left|P_{r_{j}}^{\lambda+1}\left(D_{\lambda} f\right)(\theta)\right|, \\
\text { a.e. } \quad \theta \in(0, \pi) \text { and } k \in \mathbb{N} .
\end{array}
$$


From [18, Theorem 2.2] again, we can find $C>0$ for which

$$
\left\|\max _{1 \leq j \leq k}\left|P_{r_{j}}^{\lambda} f\right|\right\|_{W_{\lambda, 1}^{p}} \leq C\|f\|_{W_{\lambda, 1}^{p}} \quad k \in \mathbb{N} .
$$

The sequence $\left\{F_{k}=\max _{1 \leq j \leq k}\left|P_{r_{j}}^{\lambda}(f)\right|\right\}_{k \in \mathbb{N}}$ is bounded in $W_{\lambda, 1}^{p}$. $W_{\lambda, 1}^{p}$, after renorming, is isometrically isomorphic to a closed subspace of $L^{p}(0, \pi) \times L^{p}(0, \pi)$. Then $W_{\lambda, 1}^{p}$, after renorming, is reflexive and the closed unit ball in $W_{\lambda, 1}^{p}$ is sequentially compact in the weak topology. Hence, there exists a subsequence $\left\{F_{k_{l}}\right\}_{l \in \mathbb{N}}$ of $\left\{F_{k}\right\}_{k \in \mathbb{N}}$ and a function $g \in W_{\lambda, 1}^{p}$ such that

$$
F_{k_{l}} \rightarrow g, \quad \text { as } \quad l \rightarrow \infty,
$$

in the weak topology of $W_{\lambda, 1}^{p}$ and $\|g\|_{W_{\lambda, 1}^{p}} \leq C\|f\|_{W_{\lambda, 1}^{p}}$. Since $F_{k_{l}}(\theta) \rightarrow$ $P_{*}^{\lambda}(f)(\theta)$, as $l \rightarrow \infty$, for $\theta \in(0, \pi)$, and $\left\{F_{k_{l}}\right\}_{l \in \mathbb{N}}$ is increasing, we conclude that $P_{*}^{\lambda}(f)=g$ and the proof of Theorem 3 is finished.

The argument in the proof of the last theorem can be used in those cases in which formulas (12) and (18) produce identities like (19). This is the case of the maximal operator associated with partial sums for ultraspherical expansions. In fact the following theorem can be proved with these ideas and $[\mathbf{9}$, Theorem F].

Theorem 4. Let $1<p<\infty$. The maximal operator $S_{*}^{\lambda}$ defined by

$$
S_{*}^{\lambda}(f)=\sup _{N \in \mathbb{N}}\left|\sum_{n=0}^{N} a_{n}^{\lambda}(f) \varphi_{n}^{\lambda}\right|
$$

is bounded from $W_{\lambda, 1}^{p}$ into itself.

Acknowledgement. The fourth author is grateful for the kind invitation to participate in this research and for the hospitality received during his visit at Universidad Autónoma de Madrid and Universidad de La Laguna.

\section{References}

[1] R. Askey And S. WAinger, A transplantation theorem between ultraspherical series, Illinois J. Math. 10 (1966), 322-344.

[2] R. Askey And S. Wainger, A transplantation theorem for ultraspherical coefficients, Pacific J. Math. 16 (1966), 393-405.

[3] B. Bongionnni and J. L. Torrea, Sobolev spaces associated to the harmonic oscillator, Proc. Indian Acad. Sci. Math. Sci. 116(3) (2006), 337-360. 
[4] B. Bongionnni and J. L. Torrea, What is a Sobolev space for the Laguerre function systems? Studia Math. 192(2) (2009), $147-172$.

[5] D. Buraczewski, T. Martínez, and J. L. Torrea, CalderónZygmund operators associated to ultraspherical expansions, Canad. J. Math. 59(6) (2007), 1223-1244.

[6] Ó. Ciaurri, A. Nowak, and K. Stempak, Jacobi transplantation revisited, Math. Z. 257(2) (2007), 355-380.

[7] G. Gasper And W. TrebeLs, Ultraspherical multipliers revisited, Acta Sci. Math. (Szeged) 60(1-2) (1995), 291-309.

[8] D. Gilbarg and N. S. Trudinger, "Elliptic partial differential equations of second order", Grundlehren der Mathematischen Wissenschaften 224, Springer-Verlag, Berlin-New York, 1977.

[9] J. E. Gilbert, Maximal theorems for some orthogonal series. I, Trans. Amer. Math. Soc. 145 (1969), 495-515.

[10] P. Graczyk, J.-J. Loeb, P. LóPez, A. Iris, A. Nowak, R. URbina, and O. Wilfredo, Higher order Riesz transforms, fractional derivatives, and Sobolev spaces for Laguerre expansions, J. Math. Pures Appl. (9) 84(3) (2005), 375-405.

[11] J. Kinnunen, The Hardy-Littlewood maximal function of a Sobolev function, Israel J. Math. 100 (1997), 117-124.

[12] J. Kinnunen and P. Lindqvist, The derivative of the maximal function, J. Reine Angew. Math. 503 (1998), 161-167.

[13] J. Kinnunen and E. Saksman, Regularity of the fractional maximal function, Bull. London Math. Soc. 35(4) (2003), 529-535.

[14] B. Muckenhoupt, Transplantation theorems and multiplier theorems for Jacobi series, Mem. Amer. Math. Soc. 64(356) (1986), iv $+86 \mathrm{pp}$.

[15] B. Muckenhoupt and E. M. Stein, Classical expansions and their relation to conjugate harmonic functions, Trans. Amer. Math. Soc. 118 (1965), 17-92.

[16] R. Radha and S. Thangavelu, Multipliers for Hermite and Laguerre Sobolev spaces, J. Anal. 12 (2004), 183-191.

[17] E. M. SteIn, "Topics in harmonic analysis related to the Littlewood-Paley theory", Annals of Mathematics Studies 63, Princeton University Press, Princeton, N.J., University of Tokyo Press, Tokyo, 1970.

[18] K. Stempak, Conjugate expansions for ultraspherical functions, Tohoku Math. J. (2) 45(4) (1993), 461-469.

[19] K. Stempak, Jacobi conjugate expansions, Studia Sci. Math. Hungar. 44(1) (2007), 117-130. 
[20] G. SzeGÖ, "Orthogonal polynomials", Fourth edition, American Mathematical Society, Colloquium Publications XXIII, American Mathematical Society, Providence, R.I., 1975.

[21] S. Thangavelu, On regularity of twisted spherical means and special Hermite expansions, Proc. Indian Acad. Sci. Math. Sci. 103(3) (1993), 303-320.

Jorge J. Betancor, Juan C. Fariña, and Lourdes Rodríguez-Mesa:

Departamento de Análisis Matemático

Universidad de la Laguna

Campus de Anchieta

Avda. Astrofísico Francisco Sánchez, s/n

38271 La Laguna (Sta. Cruz de Tenerife)

Spain

E-mail address: jbetanco@ull.es

E-mail address: jcfarina@ull.es

E-mail address: lrguez@ull.es

Ricardo Testoni:

Departamento de Matemática

Universidad Nacional del Sur

Avda. Alem 1253

8000 Bahía Blanca, Buenos Aires

Argentina

E-mail address: ricardo.testoni@uns.edu.ar

José L. Torrea:

Departamento de Matemáticas

Universidad Autónoma de Madrid

and ICMAT CSIC-UAM-UCM-UC3M

Ciudad Universitaria de Canto Blanco

28049 Madrid

Spain

E-mail address: joseluis.torrea@uam.es

Primera versió rebuda el 27 de novembre de 2008, darrera versió rebuda el 7 de maig de 2009. 